\title{
EARLY CATHETER REMOVAL AFTER ANTERIOR ANASTOMOTIC (3 DAYS) AND VENTRAL BUCCAL MUCOSAL ONLAY (7 DAYS) URETHROPLASTY
}

\author{
HOSAM S. AL-QUDAH, ANDRE G. CAVALCANTI, RICHARD A. SANTUCCI \\ Department of Urology (HSAQ, RAS), Wayne State University School of Medicine, Detroit, Michigan, USA, \\ and Section of Urology (AGC), Souza Aguiar Municipal Hospital, Rio de Janeiro, Brazil
}

\begin{abstract}
Introduction: Physicians who perform urethroplasty have varying opinions about when the urinary catheter should be removed post-operatively, but research on this subject has not yet appeared in the literature. We performed voiding cystourethrogram (VCUG) on our anterior urethroplasty patients on days 3 (anastomotic) and 7 (buccal) in an effort to determine the earliest day for removal of the urethral catheter.

Materials and Methods: Retrospective chart review of 29 urethroplasty patients from October 2002 - August 2004 was performed at two reconstructive urology centers. 17 patients had early catheter removal (12 anastomotic and 5 ventral buccal onlay urethroplasty) and were compared to 12 who had late removal (7 anastomotic and 5 buccal).

Results: Of those with early catheter removal, $2 / 12$ (17\%) of anastomotic urethroplasty patients had extravasation, which resolved by the following week and $0 / 5(0 \%)$ of the buccal mucosal urethroplasty patients had extravasation. Patients with late catheter removal underwent VCUG 6-14 days (mean 8 days) after anastomotic urethroplasty and 9-14 days (mean 12 days) after buccal mucosal urethroplasty. $0 \%$ of the anastomotic urethroplasty had leakage after the late VCUG and 1/5 (20\%) of the buccal patients had extravasation after the VCUG. Recurrences were low in all patient groups.

Conclusion: Catheter removal after anastomotic and buccal mucosal urethroplasty can be safely attempted on the 3rd and 7th post-operative days respectively, with a low rate of extravasation on VCUG. Eliminating the catheter as soon as possible should improve patient comfort without harming results and decrease the overall negative impact of surgery on the patient.
\end{abstract}

Key words: urethra; urethral stricture; surgery; catheter; device removal

Int Braz J Urol. 2005; 31: 459-64

\section{INTRODUCTION}

Urethroplasty is a common procedure performed in many centers around the world. While physicians agree on many details of the procedure, some controversy still exists about other specific details of urethroplasty patient care. The earliest feasible time to remove the catheter after surgery is a point of sig- nificant discordance in the literature. The shortest time to remove the catheter after anterior anastomotic urethroplasty seen in the literature is 7 days, and can range as high as 14 days (Table-1). The earliest time to remove the catheter reported after buccal mucosal urethroplasty is 7 days (but with concurrent suprapubic drainage for 14 days) and ranges as high as 28 days (Table-2). To our knowledge, there are no re- 
Table 1 - Reported Foley catheter removal times after anastomotic urethroplasty.

\begin{tabular}{lrccc}
\hline Reference & N & Stricture Length (cm) & $\begin{array}{c}\text { Catheter Removal } \\
\text { Time (days) }\end{array}$ & $\begin{array}{c}\text { RUG Leak } \\
\text { Rate (\%) }\end{array}$ \\
\hline Santucci et al. (2002) (6) & 168 & 1.7 & 14 & 1 \\
Micheli et al. (2002) (10) & 74 & $0.5-3$ & 7 & - \\
Jakse \& Marberger (1986) (11) & 105 & $1-4$ & 10 & 10 \\
Azoury (1976) (12) & 13 & - & 21 & - \\
\hline
\end{tabular}

Table 2 - Reported Foley catheter removal times after buccal mucosal graft onlay urethroplasty.

\begin{tabular}{lccccc}
\hline Reference & Type of Urethroplasty & $\mathbf{N}$ & $\begin{array}{c}\text { Stricture Length } \\
(\mathbf{c m})\end{array}$ & $\begin{array}{c}\text { Catheter Removal } \\
\text { Time (days) }\end{array}$ & $\begin{array}{c}\text { RUG Leak } \\
\text { Rate (\%) }\end{array}$ \\
\hline Kellner et al. (2004) (13) & Ventral & 23 & 3.7 & $14-21$ & $?$ \\
Fichtner et al. (2004) (14) & Ventral & 32 & 4.3 & $10 *$ & 6 \\
Pansadoro et al. (2003) (15) & Ventral and dorsal & 65 & 4 & $7 * *$ & $?$ \\
Heinke et al. (2003) (16) & Ventral & 38 & $?$ & 21 & $?$ \\
Kane et al. (2002)(17) & Ventral & 53 & $?$ & $14-21$ & $?$ \\
Andrich et al. (2001)(18) & Ventral and dorsal & 71 & $?$ & 21 & $?$ \\
Meneghini et al. (2001) (19) & Ventral & 20 & 2.8 & $21-28$ & $?$ \\
\hline
\end{tabular}

* Suprapubic urine diversion for 21 days, ** Suprapubic urine diversion for 14 days

ports that specifically study this parameter, and time of catheter removal remains largely a matter of physician opinion. In order to determine the earliest feasible time to remove the urethral catheter, we performed voiding cystourethrogram (VCUG) in our anterior urethroplasty patients on the third postoperative day (POD) (the 4th day if this fell on a weekend) after anastomotic urethroplasty and on the 7th POD after ventral buccal mucosal onlay urethroplasty.

There are several reasons why early catheter removal is desirable in urethroplasty patients. First, studies of patients with urethral catheters after prostate surgery have established that removal of the catheter improves both patient comfort and mobility (1). Second, there is some theoretical harm from the catheter on the just-completed delicate repair. All catheters result in some inflammatory reaction (2), with silicone catheters causing the least (but still measurable) amount in experimental studies (3). Third, in general, removing the urethral catheter earlier following surgery will decrease the total discomfort of the patient after urethroplasty. Decreasing the total im- pact of surgery makes urethroplasty a more palatable option for patients and more comparable to lesser impact surgery, such as direct visual internal urethrotomy (DVIU). DVIU has been shown not to be effective against recurrent strictures $(4,5)$, but it remains extremely popular, likely because it is so easy to perform and easy on the patient. As innovations in urethroplasty continue to decrease the impact of surgery, perhaps inappropriate persistence with DVIU, especially in those with little chance of lasting cures, will decrease.

\section{MATERIALS AND METHODS}

We performed a retrospective chart review of 32 patients with anterior urethra stricture who underwent anastomotic urethroplasty (19 patients) and ventral buccal mucosal onlay urethroplasty (10 patients) in the period from October 2002 to August 2004 at 2 referral centers for urologic reconstruction. One patient with previous local radiotherapy and 2 patients with end stage renal disease on dialysis were excluded 
from the study because of expectations for poor healing. Urethroplasty was performed by 2 surgeons (RAS, AGC). Patients were divided into 2 groups. In the early catheter removal group, we performed a VCUG on POD 3 (day 4 if it fell on a weekend) on anterior anastomotic urethroplasty patients $(\mathrm{n}=12)$ and on POD 7 on ventral buccal mucosal graft urethroplasty patients $(\mathrm{n}=5)$. In the late catheter removal group, we performed VCUG on average 8 days after surgery for anastomotic urethroplasty, and on average 12 days after surgery for buccal mucosal urethroplasty. We compared the 2 groups by the rate of leakage seen on VCUG and the recurrence rate. Patients who had extravasation in the first VCUG had replacement of the Foley catheter and a second VCUG in 7 days. Patients were followed closely after surgery for recurrence, as has been previously described (6). Review of urinary symptoms, urinary flow rates and postvoid residuals were measured $3,6,9,12$ and 24 months after surgery (7). Patients are considered to have undergone retrograde urethrogram (RUG) if obstructive voiding signs or symptoms recur after urethroplasty.

\section{RESULTS}

The average patient age was 43 years (range 19-75 years) and stricture length measured by preoperative RUG was $1.2 \mathrm{~cm}$ (range $0.5-3 \mathrm{~cm}$ ) for anasto- motic and $3.6 \mathrm{~cm}$ (range $2.5-5 \mathrm{~cm}$ ) for buccal urethroplasty. Cause of stricture was inflammatory in 8 patients, trauma in 6 , iatrogenic in 3 , and unknown in 12 patients. Patient follow-up averaged 14 months (range 3-30 months).

\section{Anastomotic Urethroplasty}

In the early group (12 patients), 2 (17\%) of the anastomotic urethroplasty patients had extravasation in the first VCUG. The other 10 patients $(83 \%)$ had a normal VCUG and the Foley removed at the same time (Figure-1). Patients with extravasation had their Foley replaced and the VCUG repeated after one week, which was normal in both cases. In the late group (7 patients), $100 \%$ of the VCUGs were normal and the patients had their Foley removed (Figure-2). There were 2 recurrences: 1 (8\%) in the early group and $1(14 \%)$ in the late group.

\section{Ventral Buccal Mucosal Graft Onlay Urethroplasty}

In the early group (5 patients), $100 \%$ had a normal VCUG and all had their Foley removed on the $7^{\text {th }}$ POD. In the late group ( 5 patients), one patient (20\%) had extravasation and the other 4 patients were normal and had their Foley removed (Figures-1 and 2 ). The second VCUG was normal and Foley removed. The recurrence rate was $0 \%$ in both groups.

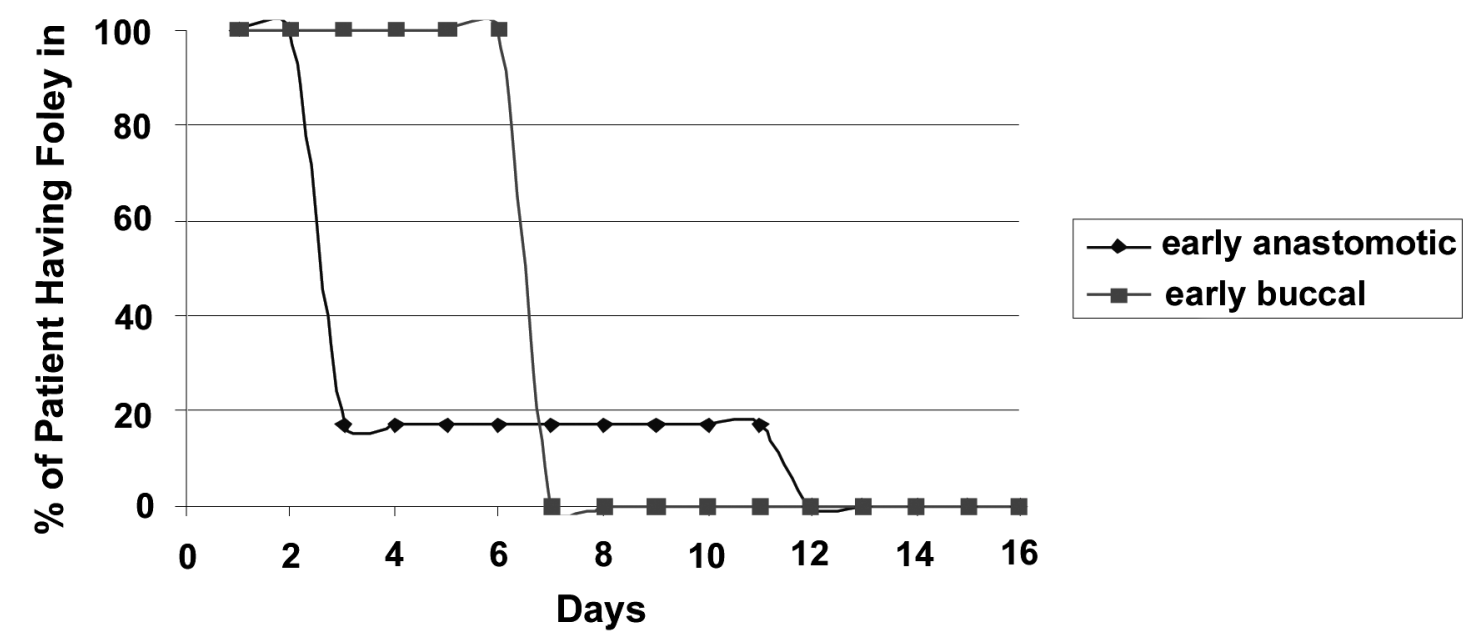

Figure 1 - Foley catheter removal times after anterior urethroplasty using an early removal protocol. 


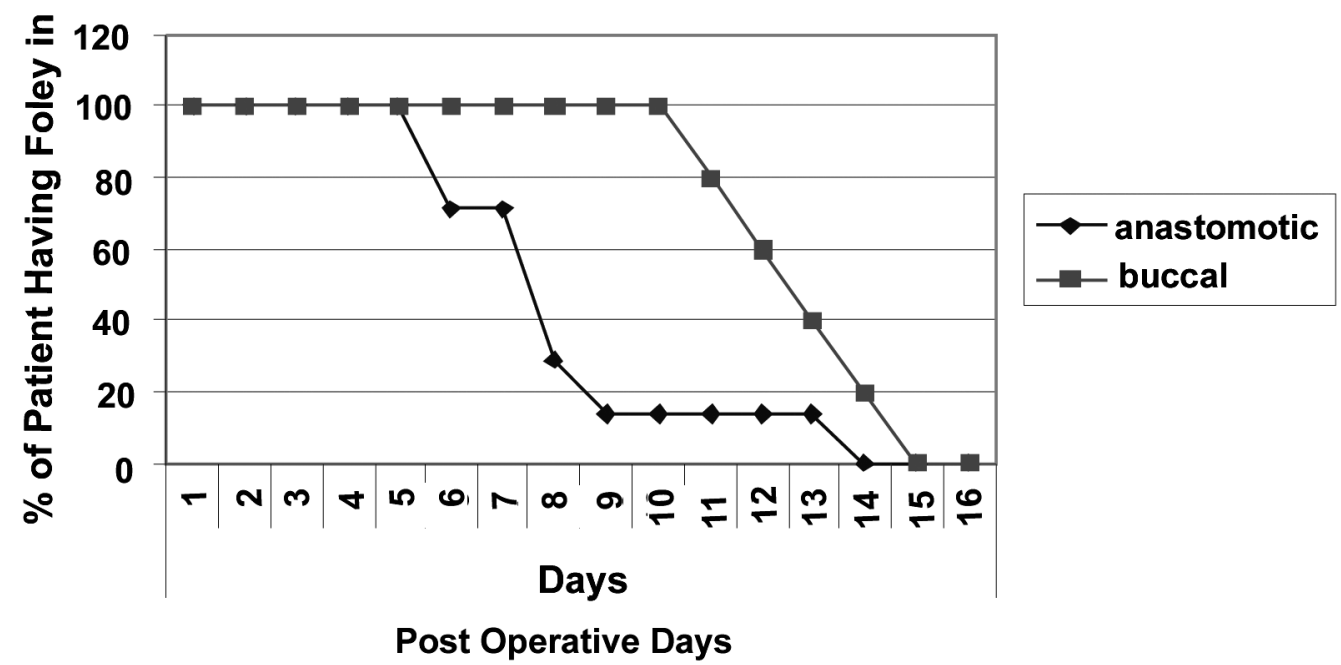

Figure 2 - Foley catheter removal times after anterior urethroplasty using a late removal protocol.

\section{COMMENTS}

The best interval period for catheterization after urethroplasty is unknown, and most published recommendations represent expert opinion only. For anastomotic urethroplasty, suggested catheterization periods range from 7 to 21 days, but only 2 reports mention the rate of extravasation when this timing is followed: $1 \%$ in 1 series and $10 \%$ in another, however this second series mixed anterior and posterior urethroplasty patients (Table-1). In our anastomotic urethroplasty patients with early catheter removal, on POD 3 there was a $17 \%$ rate of extravasation. All of these patients subsequently had catheters removed on POD 10. The stricture recurrence rate was equivalent in these 2 groups.

While this rate of leakage is higher than previously reported after longer catheterization times, it shows that over $80 \%$ of patients can have their Foley removed after anastomotic urethroplasty within 3 days time. In addition to increasing patient comfort, this approach provides some logistical benefits to the patient. In those centers that routinely admit the patient for 3-4 days postoperatively, removing the Foley catheter on POD 3 will allow the patient to be discharged without a catheter. This will improve patient comfort, eliminate troublesome catheter care at home and also allow out-of-town patients to return home without needing further acute follow-up.

For buccal mucosal graft ventral onlay urethroplasty, recommended catheterization periods range from 14 to 28 days (Table-2). In 2 series, the Foley is removed on POD 7 and POD 10, but urine is diverted suprapubically for another 7-14 days. When we removed the Foley on POD 7 after buccal mucosal urethroplasty, no patient in our series had urinary extravasation. One patient in the late Foley removal group had leakage, and the Foley was removed 7 days later after a normal VCUG was obtained. The stricture recurrence rate was equivalent whether or not the Foley was removed early or late.

Prolonged catheterization is consistently reported by our patients and those of other physicians $(1,8)$ as the most troublesome part of their surgery experience, and we believe that limiting this unpleasant experience significantly decreases the negative "impact" of surgery. As we learn to decrease the requirements for postoperative stay, in some cases using same-day surgery (9), then an anastomotic urethroplasty becomes more comparable to minimally invasive therapies like direct visual internal urethrotomy (DVIU) with its attendant short catheter times (usually 3 days), and thus potentially more acceptable to both patient and surgeon. 


\section{CONCLUSION}

Catheter removal after anastomotic and buccal mucosal urethroplasty can be safely attempted on the $3 \mathrm{rd}$ and 7 th postoperative days respectively, with a low rate of extravasation on VCUG. Getting the Foley out earlier did not increase recurrence rates on short-term follow-up. Eliminating the catheter as soon as possible has beneficial effects on patient comfort and the overall negative "impact" of surgery.

\section{REFERENCES}

1. DeMarco RT, Bihrle R, Foster RS: Early catheter removal following radical retropubic prostatectomy. Semin Urol Oncol. 2000; 18: 57-9.

2. Ruutu ML, Talja MT, Andersson LC, Alfthan OS: Biocompatibility of urinary catheters-present status. Scand J Urol Nephrol Suppl. 1991; 138: 235-8.

3. Talja M, Korpela A, Jarvi K: Comparison of urethral reaction to full silicone, hydrogen-coated and siliconised latex catheters. Br J Urol. 1990; 66: 652-7.

4. Pansadoro V, Emiliozzi P: Internal urethrotomy in the management of anterior urethral strictures: long-term followup. J Urol. 1996; 156: 73-5.

5. Heyns CF, Steenkamp JW, De Kock ML, Whitaker P: Treatment of male urethral strictures: is repeated dilation or internal urethrotomy useful? J Urol. 1998; 160: 356-8.

6. Santucci RA, Mario LA, McAninch JW: Anastomotic urethroplasty for bulbar urethral stricture: analysis of 168 patients. J Urol. 2002; 167: 1715-9.

7. Heyns CF, Marais DC: Prospective evaluation of the American Urological Association symptom index and peak urinary flow rate for the followup of men with known urethral stricture disease. J Urol. 2002; 168: 2051-4.

8. Lepor H, Nieder AM, Fraiman MC: Early removal of urinary catheter after radical retropubic prostatectomy is both feasible and desirable. Urology. 2001; 58: 425-9.

9. Santucci RA, MacDonald M: Decreasing length of stay to 0 days after simple and complex urethroplasty. BJU Int. 2004; 94: (Abst \# UP 2.11), 167.

10. Micheli E, Ranieri A, Peracchia G, Lembo A: End-toend urethroplasty: long-term results. BJU Int. 2002; 90: 68-71.

11. Jakse G, Marberger H: Excisional repair of urethral stricture. Follow-up of 90 patients. Urology. 1986; 27 : 233-6.
12. Azoury BS, Freiha FS: Excision of urethral stricture and end to end anastomosis. Urology. 1976; 8: 13840.

13. Kellner DS, Fracchia JA, Armenakas NA: Ventral onlay buccal mucosal grafts for anterior urethral strictures: long-term followup. J Urol. 2004; 171: 726-9.

14. Fichtner J, Filipas D, Fisch M, Hohenfellner R, Thuroff JW: Long-term outcome of ventral buccal mucosa onlay graft urethroplasty for urethral stricture repair. Urology. 2004; 64: 648-50.

15. Pansadoro V, Emiliozzi P, Gaffi M, Scarpone P, DePaula F, Pizzo M: Buccal mucosa urethroplasty in the treatment of bulbar urethral strictures. Urology. 2003; 61: 1008-10.

16. Heinke T, Gerharz EW, Bonfig R, Riedmiller H: Ventral onlay urethroplasty using buccal mucosa for complex stricture repair. Urology. 2003; 61: 1004-7.

17. Kane CJ, Tarman GJ, Summerton DJ, Buchmann CE, Ward JF, O'Reilly KJ, et al.: Multi-institutional experience with buccal mucosa onlay urethroplasty for bulbar urethral reconstruction. J Urol. 2002; 167: 13147.

18. Andrich DE, Leach CJ, Mundy AR: The Barbagli procedure gives the best results for patch urethroplasty of the bulbar urethra. BJU Int. 2001; 88: 385-9.

19. Meneghini A, Cacciola A, Cavarretta L, Abatangelo G, Ferrarrese P, Tasca A: Bulbar urethral stricture repair with buccal mucosa graft urethroplasty. Eur Urol. 2001; 39: 264-7.

Received: July 8, 2005 Accepted: August 15, 2005

\author{
Correspondence address: \\ Dr. Richard A. Santucci \\ Chief of Urology, Detroit Receiving Hospital \\ 4160 John R. Suite 1017 \\ Detroit, MI, 48201, USA \\ Fax: + 1 313-745-0464 \\ E-mail:rsantucc@med.wayne.edu
}




\section{EDITORIAL COMMENT}

In spite of the great advances in urethral reconstructive surgery, there are still many controversies. Considerable controversy is related to the urinary drainage after urethroplasty. This generates many questions: using of intra-urethral or suprapubic catheter, or both; how long and by whom; which size (12-20 Ch) and type of the catheter? According to the different authors, duration of urinary drainage ranges from 1-3 weeks. There is also suprapubic drainage with small fenestrated or grooved stents, which only pass the level of urethral repair. There is not unique approach, regarding urinary drainage after urethroplasty - it depends on the type of urethroplasty and surgeon's preference. There is no comparable study in the present literature.

In this present study, the authors evaluated early removal of intraurethral catheter after anterior anastomotic and ventral buccal mucosa onlay urethroplasty on the third and seventh days,

\section{EDITORIAL COMMENT}

For some years ago, I used to remove the urethral catheter 3 to 5 days after ventral urethroplasty and 7 to 10 days after ventral buccal onlay urethroplasty with good results. respectively. They had control group of patients with the same types of urethroplasty with late removal of the catheter on eight and twelfth days, respectively. Outcome was the same in both groups. Early versus late catheter removal has several advantages: catheter in native urethra may cause significant morbidity, such as irritative symptoms and discomfort, may interfere with urethral secretion and ejaculation that may be the cause of infection. However, the most important question is by whom early catheter removal should be done. Only very experienced and skillful surgeons with referral population and familiar with this demanding field of surgery can achieve such a success, as in this study. These results could hardly be reproduced by the surgeon who performs urethroplasty occasionally. Early catheter removal after urethroplasty is intriguing, but limited on relatively small number of the patients with short-term results. Long-term results are awaited with great interest.

\author{
Dr. Sava V. Perovic \\ Professor of Urology/Surgery \\ School of Medicine, University of Belgrade \\ Belgrade, Serbia and Montenegro \\ E-mail:perovics@eunet.yu
}

I began this technique after an intentionally early removal of urethral catheter in 2 patients and results were very satisfactory.

Dr. Mostafa A. Al-Rifaei

Department of Urology

Faculty of Medicine, University of Alexandria

Alexandria, Egypt

E-mail: emadphoto@yahoo.com 